\title{
Atividade da catalase no pulmão, rim e intestino delgado não isquemiado de ratos após reperfusão intestinal
}

\author{
Catalase activity in lung, kidney and small bowel non-ischemic in rats after \\ intestinal reperfusion
}

Camila de Oliveira Ferro ${ }^{1}$; Vera lucia Antunes Chagas²; Marcus Fernandes de Oliveira ${ }^{3}$; Pedro Lagerblad de Oliveira 4 ; Alberto SCHANAIDER, TCBC-RJ

\section{R E S U M O}

\begin{abstract}
Objetivo: Avaliar a atividade catalase, após lesão por isquemia e reperfusão intestinal e estudar as alterações deste antioxidante em órgãos situados à distância do insulto inicial. Métodos: Utilizaram-se 18 ratos do tipo Wistar, aleatoriamente distribuídos em três grupos. 1-Controle, 2-Simulação e 3-Isquemia/Reperfusão. Neste último, realizou-se isquemia no íleo, por 60 minutos, seguida de reperfusão por 30 minutos. No grupo 2 efetuou-se apenas uma laparotomia. Foram retirados, de todos os animais, segmentos do intestino com e sem reperfusão, além do pulmão e rim direitos para exame com microscopia óptica. A atividade da catalase foi aferida em espectrofotômetro ajustado para $240 \mathrm{~nm}$. Utilizaram-se os testes estatísticos Mann e Whitney e Kruskal Wallis. Resultados: Observou-se aumento significante $(p<0.05)$, da atividade da catalase nas porções do intestino isquemiado e não isquemiado, além do pulmão. Houve redução da atividade enzimática no rim. No grupo com reperfusão observaram-se alteração nas vilosidades, infiltrado inflamatório em todas as vísceras, além de áreas de atelectasia pulmonar. Conclusão: O estresse oxidativo intestinal, em ratos, causa alterações bioquímicas à distância com mobilização dos mecanismos de defesa antioxidante pulmonar, em segmento intestinal não isquemiado e no rim, com esgotamento precoce das reservas deste último, no entanto, sem lesão celular relevante, destas vísceras.
\end{abstract}

Descritores: Intestino delgado. Catalase. Pulmão. Rim. Ratos.

\section{INTRODUÇÃO}

Un'sers m dos mecanismos orgânicos de maior impacto para a hemostasia celular é o estresse oxidativo e a produção de seus radicais. Ele é decorrente de dois eventos fisiopatológicos principais: a isquemia seguida de reperfusão e os processos inflamatórios. O estresse pode causar alterações locais e ou sistêmicas, principalmente pela liberação de substâncias citotóxicas (radicais livres derivados do oxigênio entre outras), oriundas da cadeia respiratória, ou de neutrófilos polimorfonucleares e de células endoteliais'.

Radicais livres derivados do oxigênio (RLDO) são substâncias que apresentam um elétron não emparelhado em seu orbital externo, o que gera forte carga destrutiva de diversos componentes celulares quando produzidos em excesso, ou quando suplantam mecanismos orgânicos de defesa ${ }^{2}$. A cadeia respiratória produz estes radicais em consequência de processos metabólicos normais, mas na vigência de um estresse oxidativo, a exemplo dos processos de isquemia e reperfusão (I/R) ou inflamatórios, há explosão de síntese destas espécies reativas do oxigênio. A isquemia cria condições para que, no momento da reperfusão, enzimas ativadas participem da redução do oxigênio molecular gerando oxirradicais.

Os radicais livres derivados do oxigênio apesar de agirem contra microorganismos agressores, também causam lesões celulares graves, se produzidos em grandes quantidades em face da manutenção do agente causal. Os principais radicais são o anionte superóxido $\left(\mathrm{O}_{2}^{-}\right)$, o peróxido de hidrogênio $\left(\mathrm{H}_{2} \mathrm{O}_{2}\right)$ e o radical hidroxila $\left(\mathrm{OH}^{-}\right)$. Quando em excesso promovem ruptura de membranas celulares, lise nuclear, entre outros efeitos deletérios, mas também têm a capacidade de ativar substâncias vasomotoras e próinflamatórias, vinculadas ao aumento de fatores da transcrição nuclear e com repercussões locais e sistêmicas ${ }^{3}$.

Apesar do potencial destrutivo dos RLDO, as células têm mecanismos de defesa que bloqueiam ou limitam a ação destes e que incluem sistemas enzimáticos intracelulares, denominados como inativadores, bloqueadores ou varredores. Há um sistema de proteção antioxidante formado por enzimas e compostos de baixo peso molecular, situados nas membranas de organelas, ou

Trabalho realizado no Programa de Pós-Graduação do Departamento de Cirurgia - FM/UFRJ - Rio de Janeiro - RJ-BR.

1. Mestranda do Departamento de Cirurgia da Faculdade de Medicina da Universidade Federal do Rio de Janeiro (UFRJ) - Rio de Janeiro - RJ-BR. e Médica Veterinária. 2. Professora Adjunta do Departamento de Patologia FM/UFRJ- Rio de Janeiro - RJ-BR. 3. Professor Adjunto do Instituto de Bioquímica Médica da UFRJ- Rio de Janeiro - RJ-BR. 4. Professor Titular do Instituto de Bioquímica Médica da UFRJ- Rio de Janeiro - RJ-BR. 5. Coordenador do Programa de Pós-Graduação do Departamento de Cirurgia - FM/UFRJ- Rio de Janeiro - RJ-BR. 
no meio intracelular. Dentre estes sobressai a catalase, localizada na mitocôndria e peroxissomas, exceto nos eritrócitos, onde é encontrada no citosol. Ela é responsável pela inativação, no interior da célula, do peróxido de hidrogênio em água, situação esta que impede a cascata de formação de novos subprodutos decorrentes da sucessiva redução monovalente destes intermediários oxidantes.

Uma produção excessiva destes radicais, oriunda de processo patológico persistente, pode suplantar tais mecanismos e causar lesão tecidual direta, peroxidação lipídica das membranas celulares, carbonilação das proteínas, citoesqueletólise e alterações do DNA. Paradoxalmente, a reperfusão, embora seja necessária para reverter o estado isquêmico, piora as lesões já presentes no período de isquemia quando ainda não se instalou a necrose tecidual ${ }^{4,5}$

$A$ isquemia seguida da reperfusão (I/R) pode ser considerada um evento comum à diversas doenças da prática clínico-cirúrgica. Quando o processo se instala, há liberação intensa dos oxirradicais local e de outros mediadores humorais e que pode comprometer a estrutura e o funcionamento celular, com impacto em várias vísceras e à distância.

A isquemia mesentérica é uma das doenças mais graves do trato gastrintestinal e, dependendo do seu tempo de evolução, o processo pode evoluir para necrose intestinal ao mesmo tempo em que, quando restabelecido o fluxo sanguíneo, ele pode agravar os danos ocorridos na fase isquêmica através da formação dos radicais livres do oxigênio. A lesão da mucosa intestinal proveniente da isquemia e reperfusão é bem conhecida, mas muito pouco há acerca do comprometimento de porções do sistema digestório, à distância do foco da lesão primária ${ }^{6,7}$.

Da mesma forma, lesões pulmonares graves podem decorrer da geração de metabólitos tóxicos do oxigênio em quantidade que exceda a capacidade antioxidante deste órgão. Poucos estudos avaliaram o estresse oxidativo pulmonar em modelos experimentais de agressão tecidual sistêmica ${ }^{8-11}$.

No rim, a lesão por reperfusão também é causada pelas espécies reativas do oxigênio e a peroxidação lipídica com participação da xantina oxidase e da nicotinamida adenina dinucleotídio fosfato (NADPH) e modelos de insuficiência renal comprovam tais ações ${ }^{12,13}$.

Ainda não são bem conhecidos os mecanismos fisiopatológicos relacionados às lesões à distância de segmentos intestinais isquemiados.

O presente estudo teve como principal objetivo elucidar melhor a fisiopatologia do estresse oxidativo proveniente da isquemia e reperfusão intestinal e suas possíveis repercussões à distância, em segmento intestinal não isquemiado, no pulmão e rim, através da dosagem da enzima antioxidante catalase e de estudos histológicos destes órgãos.

\section{MÉTODOS}

Foi realizado um estudo experimental com a utilização de 18 ratos da linhagem Wistar - Rattus norvegicus, pesando aproximadamente 250 g, com condições controladas de temperatura e jejum para sólidos de 48 horas. Os procedimentos foram aprovados pela Comissão de Ética ao Uso de Animais de Experimentação da Faculdade de Medicina Universidade Federal do Rio de Janeiro.

Os animais foram anestesiados com cloridrato de cetamina na concentração de $10 \mathrm{mg} / 100 \mathrm{~g}$ e dose de 0,2 $\mathrm{mL} / 100 \mathrm{~g}$ e cloridrato de xilazina na concentração de 1 $\mathrm{mg} / 100 \mathrm{~g}$ na dose de 0,05 mL/100 g via intraperitoneal. Os animais foram distribuídos em três grupos: Controle (C), Simulação (S) e Isquemia e Reperfusão (I/R).

$\mathrm{O}$ acesso cirúrgico à cavidade abdominal em todos, consistiu de uma incisão mediana. No grupo I/R procedeu-se isquemia em um segmento ileal, distando $5 \mathrm{~cm}$ da válvula íleo-cecal, mediante a colocação de um microclampe em um ramo da artéria mesentérica superior associada à colocação de outros dois microclampes na arcada marginal com $5 \mathrm{~cm}$ de distância entre si. A isquemia teve duração de 60 minutos, seguida de reperfusão por 30 minutos. Ao término da reperfusão foram retirados $5 \mathrm{~cm}$ do segmento do intestino submetido à isquemia e reperfusão, assim como um fragmento de $5 \mathrm{~cm}$ de íleo não reperfundido situado a $3 \mathrm{~cm}$ de distância da margem isquêmica, além de pequena amostra do rim direito. Em todos os animais, ainda no mesmo ato operatório, efetuou-se uma toracotomia direita com a exérese de $0,5 \mathrm{~cm}$ do lobo inferior do pulmão direito. No grupo S houve apenas manipulação intestinal e exposição da cavidade por 90 minutos, após o que procedeuse a coleta das amostras, à semelhança do grupo I/R. Do grupo $C$ apenas foram obtidos fragmentos de íleo, rim e pulmão. Todas as amostras foram divididas, imediatamente após a coleta. A parte destinada a avaliação da atividade da catalase em espectrofotômetro foi colocada em eppendorfs, congelada em nitrogênio líquido e estocada no freezer a $-70^{\circ} \mathrm{C}$. A outra metade foi fixada em formol a $10 \%$ e enviada para processamento histológico. Este preparado foi corado pela hematoxilina e eosina (HE) e analisado em microscópio óptico Ortholux, com aumentos de 100, 250 e 400 vezes.

\section{Preparo das amostras de tecidos para a leitura da atividade da enzima catalase}

Após descongelamento em temperatura ambiente, foi realizado um homogenato de cada amostra dos tecidos contendo coquetel de inibidores de proteases, sendo eles PMSF 100 mM - inibidor de serinoprotease, EDTA $100 \mathrm{mM}$ - inibidor de metaloprotease e SBTI $10 \mathrm{mg}$ - inibidor de tripsina e solução tampão de fosfato de potássio $50 \mathrm{Mm}$ em $\mathrm{pH} 7,0$. O volume de $2 \mathrm{~mL}$ de cada amostra foi colocado em eppendorfs e centrifugado a $12.000 \mathrm{rpm}$, durante 10 minutos, em temperatura de $4^{\circ} \mathrm{C}$. Terminada a centrifugação foram constituídas quatro alíquotas do sobrenadante de $200 \mu \mathrm{l}$ de cada amostra homogeneizada e após, estocadas em $-70^{\circ} \mathrm{C}$.

\section{Leitura da atividade da catalase}

Ela foi feita em espectrofotômetro modelo GBC 920 com comprimento de onda ajustado para 240 nm, es- 
pecífico para a enzima em questão. Utilizou-se tampão catalase contendo fosfato de potássio $50 \mathrm{mM}+$ EDTA 0,1 $m M+$ Triton X-100 0,002\%. O peróxido de hidrogênio $\left(\mathrm{H}_{2} \mathrm{O}_{2}\right) 300 \mathrm{mM}$ foi adicionado para disparar a reação junto a 10 ul da amostra.

\section{Avaliação estatística}

Para análises estatísticas da catalase foram utilizados os testes de Mann e Whitney, e de Kruskal Wallis, respectivamente, para a comparação entre dois ou mais de dois grupos. Utilizou-se o software Prisma (versão 4.03). Os valores foram considerados significantes para um intervalo de confiança de $95 \%(p<0,05)$.

\section{RESULTADOS}

Atividade enzimática da catalase, intestinal, pulmonar e renal

Observou-se aumento da atividade catalásica no intestino isquemiado e na porção proximal não isquemiada (Figura 1), assim como no pulmão (Figura 2). Não houve diferenças significantes na comparação do grupo Simulação com o grupo Controle. Já no rim (Figura 3), verificou-se redução da atividade enzimática do grupo reperfusão ( $p=$ 0.0022 ) se comparada àquela dos grupos Simulação e Controle.

\section{Histopatologia intestinal, pulmonar e re-}

nal:

Observou-se no íleo reperfundido desarranjo leve da arquitetura das vilosidades com congestão vascular e infiltrado polimorfonuclear (Figura 4). Estas alterações foram um pouco mais tênues na porção ileal proximal, não

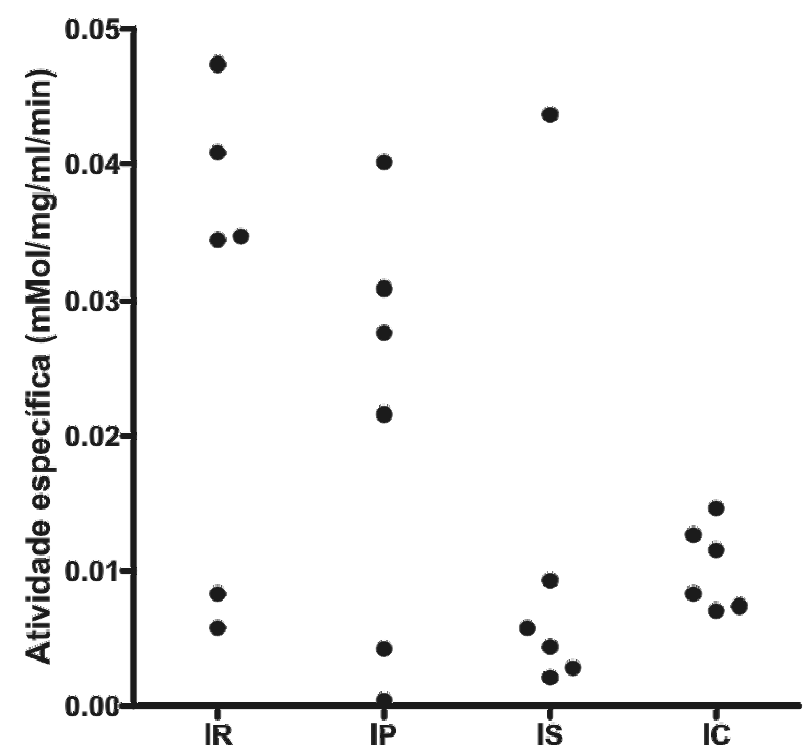

Figura 1 - Atividade da catalase no intestino com reperfusão (IR), no intestino proximal sem reperfusão (IP) e no intestino dos grupos Simulação (IS) e Controle (IC). isquemiada, com presença de arquitetura vilositária preservada, porém com leve congestão vascular e infiltrado polimorfonuclear.

Nas amostras do lobo inferior do pulmão direito, do grupo I/R constou-se congestão de septos alveolares associada a áreas de atelectasias (Figura 5).

No grupo I/R, constatou-se congestão glomerular nas amostras do rim direito (Figura 6).

\section{DISCUSSÃO}

Existem diversos trabalhos que avaliam a ação de espécies reativas do oxigênio, porém os efeitos à distân-

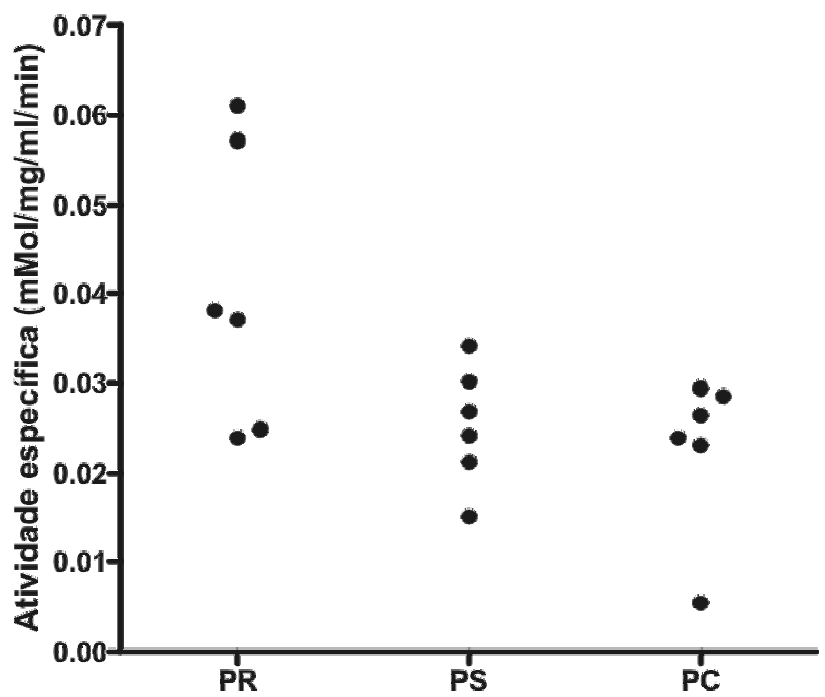

Figura 2 - $\quad$ Atividade da catalase no pulmão com reperfusão (PR), nos grupos Simulação (PS) e Controle (PC).

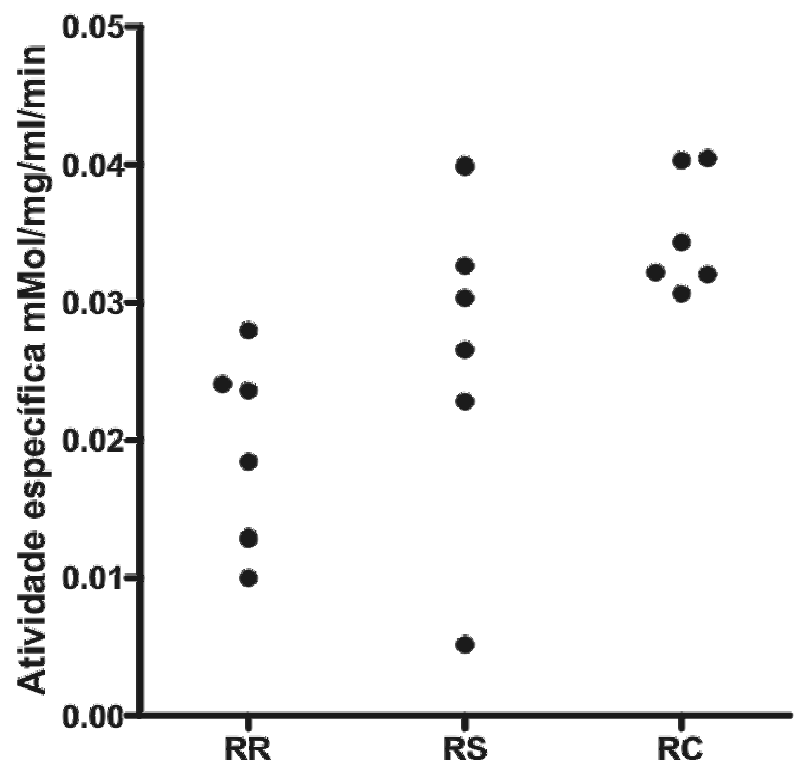

Figura 3 - $\quad$ Atividade da catalase no rim com reperfusão (RR), nos grupos Simulação (RS) e Controle (RC). 


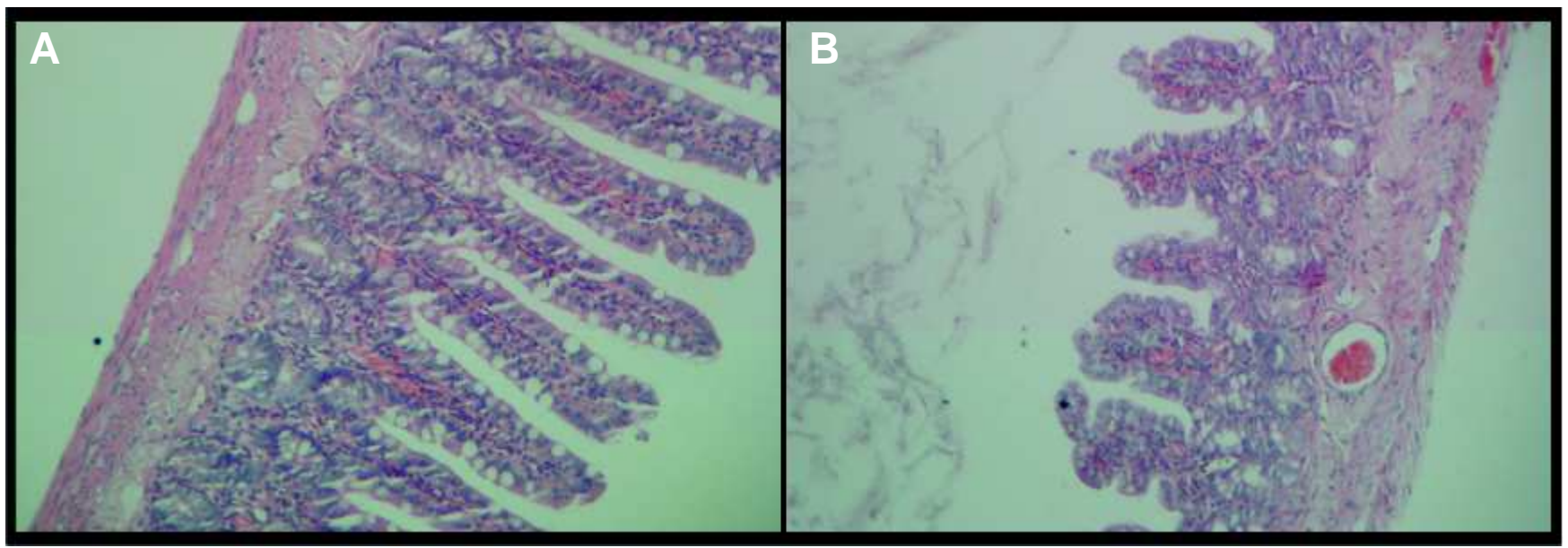

Figura 4 - A - Grupo Controle: observa-se arquitetura vilositária preservada; B - Grupo I/R: verificam-se as presenças de infiltrado polimorfonuclear da lâmina própria, desarranjo leve da arquitetura das vilosidades, além de congestão vascular. Coloração HE $x 100$

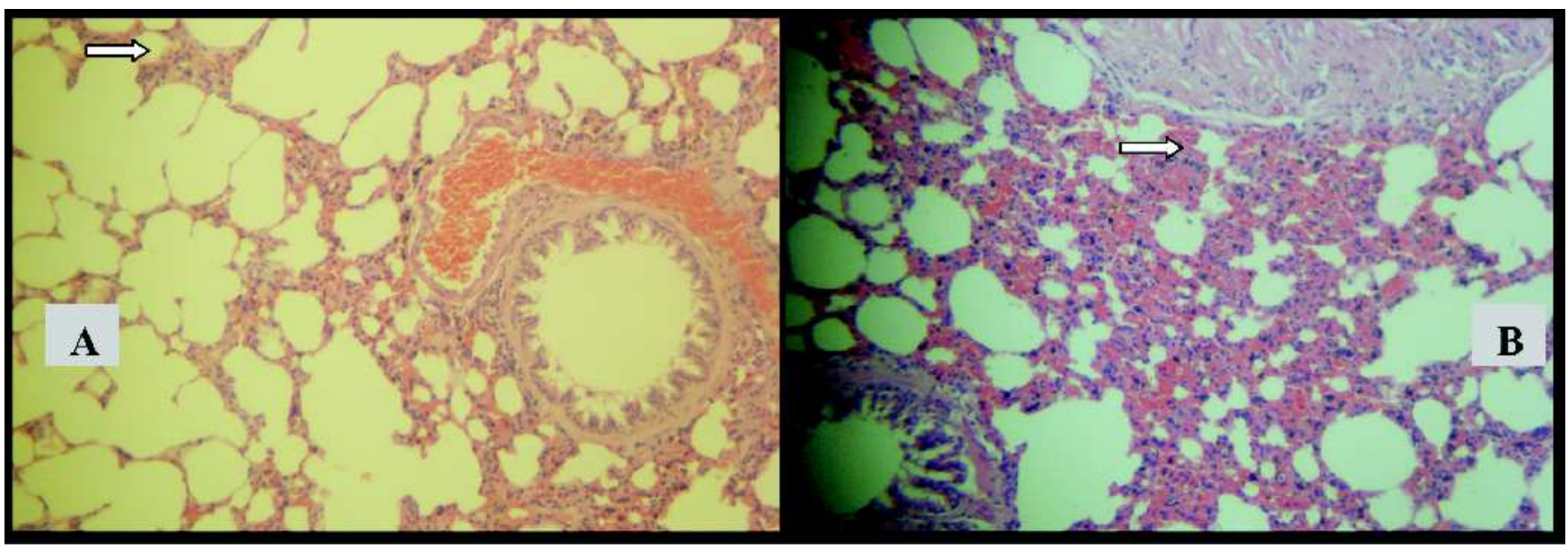

Figura 5 - A - Grupo Controle: observam-se alvéolos com parede delgada (seta) e pequena congestão vascular; B - Grupo Isquemial Reperfusão: presença de infiltrado polimorfonuclear, acentuada congestão nos septos alveolares, além de áreas de atelectasia (seta). Coloração HE x250.

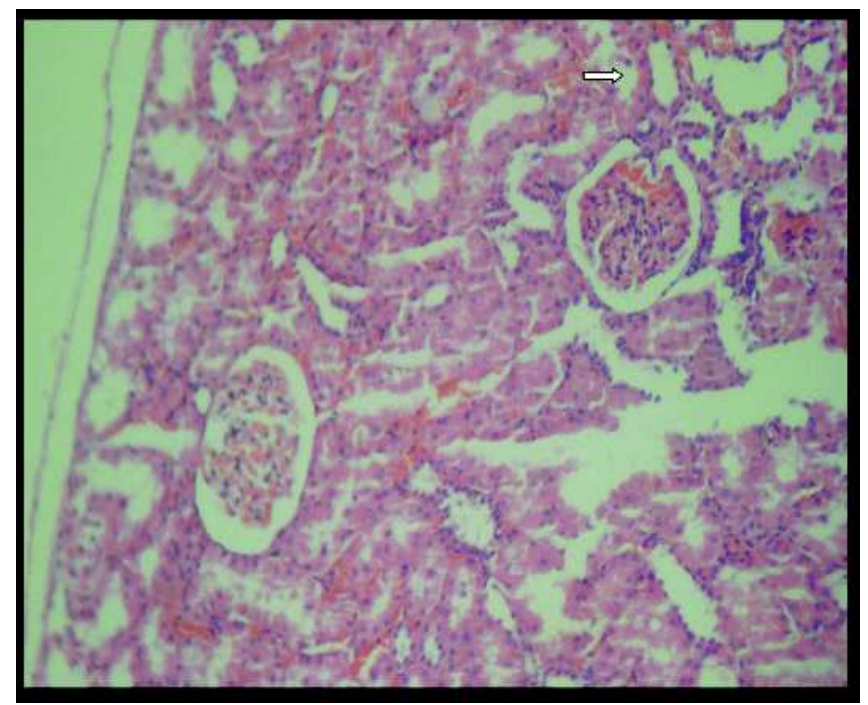

Figura 6 - Tecido renal após isquemia e reperfusão intestinal. Constata-se (seta) a presença de congestão glomerular. Coloração HE x250. cia ainda são pouco conclusivos e por vezes contraditórios. Procurou-se, portanto, investigar uma das facetas relacionadas aos antioxidantes, com ênfase a catalase e as alterações que ocorrem após isquemia seguida da reperfusão intestinal, não só em outros órgãos, mas no próprio sistema digestório, em um segmento não isquemiado.

A doença vascular intestinal representa problema clínico-cirúrgico grave, pelas elevadas taxas de morbimortalidade, apesar de todo o aprimoramento científico atual. O esclarecimento acurado dos mecanismos fisiopatológicos locais e sistêmicos, envolvidos neste processo mórbido, poderá contribuir para melhor abordagem terapêutica e melhor prognóstico ${ }^{14,15}$.

$O$ rato foi escolhido como modelo, pois apresenta vantagens da semelhança genética, o baixo custo, a possibilidade de utilização de animais isogênicos , SPF (livre de germes patogênicos) criado no Centro de Cirurgia Experimental do Departamento de Cirurgia da FM/UFRJ. Aduz-se a familiaridade do patologista com análise de teci- 
dos nesta espécie. Os animais foram distribuídos, randomicamente, em três grupos, sendo eles o Controle (C), utilizado na determinação do padrão de normalidade para a viabilidade celular e enzimática e estudo histológico; o grupo Simulação (S), no qual foi realizada a laparotomia mediana e apenas manipulação visceral; e o grupo Isquemia/ Reperfusão (I/R) intestinal, submetido ao procedimento cirúrgico. O número de animais em cada grupo foi autorizado pelo Comitê de Ética considerando a prerrogativa de utilizar o menor número capaz de determinar resultados significantes para o tipo de estudo, condição esta considerada, também, suficiente para avaliação estatística.

A escolha pela isquemia de 60 minutos baseouse em linha de pesquisa desenvolvida por um dos autores e cuja técnica já foi bem consolidada ${ }^{16}$. A confirmação clínica da isquemia se deu pela inspeção peroperatória, sendo constatada a interrupção do fluxo sanguíneo mesentérico em face do clampeamento vascular, a ausência de batimentos (pulsação) nas arcadas alvo, a discreta cianose das alças e ausência de peristalse. Já a reperfusão, do mesmo segmento, com duração de 30 minutos, foi confirmada pelo retorno do fluxo com batimentos visíveis, coloração rósea e peristalse ativa.

Os radicais livres derivados de oxigênio (RLDO) estão relacionados a diversas doenças graves, incluindo lesões por isquemia e reperfusão (I/R) e inflamatórias ${ }^{17} \mathrm{com}$ o comprometimento de diversos órgãos e sistemas, a exemplo do sistema digestório, do coração, do cérebro, dos rins e dos pulmões ${ }^{18}$.

A reperfusão em tecidos isquêmicos produz efeito paradoxal de agravamento das lesões preexistentes. 0 dano pode ser local ou sistêmico e causado pela liberação de diversos subprodutos do estresse oxidativo, bem como de outros mediadores humorais. Trata-se de reação complexa, explosiva cuja peroxidação das membranas e a carbonilação de proteínas ${ }^{15}$ promovem o desarranjo das estruturas celulares (membranas, citoesqueleto, núcleo) e comprometimento da respiração mitocondrial e da geração energética ${ }^{19}$.

Obviamente, quanto maior o período de isquemia, maiores serão as alterações celulares irreversíveis. Durante a isquemia ocorre a degradação da adenina trifosfato, o aumento da atividade da fosfolipase A2, o influxo de $\mathrm{Ca}_{2}{ }^{+}$intracelular e a conversão da xantina desidrogenase na forma oxidase. Na reperfusão, o oxigênio, ao passar na cadeia mitocondrial de transporte de elétrons, capta um elétron com a participação da nicotamida adenina dinucleotídio hidrogenase (NADH). A partir de então, em uma sequência de sucessivas reduções, novos oxirradicais são formados. O peróxido de hidrogênio origina-se da inativação do anionte superóxido pela superóxido dismutase e para que também seja inativado, com a formação de água, participam a catalase ou o sistema glutatião.

A catalase é enzima presente na maioria dos peroxissomas dos mamíferos. É molécula com quatro cadeias de polipeptídios idênticas, cada uma composta por mais de 500 aminoácidos em subunidades, com grupos heme porfirínicos e NADPH. Em altas concentrações remove $\mathrm{O}_{2} \mathrm{O}_{2}$ e catalisa a formação de água e oxigênio molecular.

Lesões vasculares intestinais com eventos isquêmicos associados à reperfusão causam problemas sérios, muitas vezes impondo intervenções cirúrgicas de urgência ${ }^{20}$. As causas principais são as tromboses mesentéricas e embolias, o encarceramento herniário, o volvo, as invaginações, ou as alterações circulatórias sistêmicas. A isquemia e reperfusão mesentéricas estão associadas à produção de espécies reativas do oxigênio. A lesão da mucosa intestinal e da barreira que a protege ocorre por ação direta sobre a citosol e núcleo ou pela degradação dos ácidos graxos polinsaturados das membranas. Isto desencadeia a migração de endotoxinas bacterianas pela circulação 21,22 . A produção de citocinas, com o aumento dos níveis plasmáticos do Fator de Necrose Tumoral (TNF) de 120 minutos foi descrito em ratos com isquemia intestinal ${ }^{21}$. A oclusão da artéria mesentérica superior por 45 minutos seguida de duas horas de reperfusão, também mostrou elevação do fator de necrose tumoral em concentrações duas vezes maiores do que após a reperfusão ${ }^{23}$.

No intestino do rato, a peroxidação lipídica está envolvida na renovação dos enterócitos, principalmente no topo das vilosidades. As células da cripta apresentam grande atividade da catalase e da superóxido dismutase (SOD), ao contrário do que se verifica no topo das vilosidades. Com isto os processos de divisão, diferenciação e migração de enterócitos ficam comprometidos. Células apoptóticas são geralmente observadas no topo das vilosidades em função de sua substituição a cada dois a três dias, mas a ação de oxirradicais promove acentuado aumento do dano tecidual, com descamação e desarranjo das vilosidades, além de causar apoptose de células situadas na cripta em uma escala cuja recomposição é insuficiente para a manutenção da integridade mucosa ${ }^{6}$.

A mucosa intestinal, principalmente do intestino delgado, é a mais gravemente afetada. Em processos isquêmicos ocorre a redução da quantidade de oxigênio que chega à extremidade das vilosidades ocorrendo lesões, inicialmente no topo destas estruturas. Sobrevém o aumento da permeabilidade vascular com edema intersticial e congestão vascular. Normalmente, a isquemia com mais de uma hora de duração seguida de reperfusão pode produzir edema de submucosa, desprendimento da mucosa, ulcerações e sangramento das vilosidades intestinais. No intestino de ratos a completa conversão da xantina desidrogenase a xantina oxidase, pré-requisito na formação de precursores do estresse oxidativo, leva aproximadamente um minuto após indução da isquemia ${ }^{21}$. O edema da mucosa intestinal e infiltração inflamatória da lâmina própria são importantes lesões observadas ${ }^{24}$, à semelhança de alguns dos achados descritos.

Isquemia intestinal causada por volvo ou oclusão vascular mesentérica tratadas com êxito, ao promoverem a reperfusão tecidual, também albergam o potencial de produção de espécies ativas do oxigênio em quantidade suficiente para causar danos à distância. 
Modelos experimentais e ensaios clínicos com observação de doenças em anima nobile demonstram a associação entre reperfusão e resposta inflamatória pulmonar e que resultam em produção crescente de radicais livres derivados do oxigênio, ambos envolvidos na patogênese da insuficiência respiratória. Tais aspectos estão relacionados aos achados no presente estudo e que evidenciou aumento de polimorfonucleares e da atividade antioxidante.

Descrições anteriores mostram que as características das lesões agudas do pulmão em modelos de l/R intestinal de ratos incluem o sequestro de neutrófilos, a redução do trifosfato de adenosina (ATP) no tecido pulmonar, o rompimento de células endoteliais alveolares e o aumento da permeabilidade vascular ${ }^{22}$. A microvasculatura pulmonar, em especial, também parece ser bastante susceptível à lesões resultantes da ativação de mediadores inflamatórios sistêmicos decorrentes de estresse oxidativo em órgãos à distância ${ }^{25}$. Estudos têm demonstrado que a delicada estrutura alvéolo-capilar é sensível à lesão de isquemiareperfusão mesmo na ausência de hipóxia; porém, poucas investigações avaliaram o estresse oxidativo pulmonar em modelos experimentais de agressão tecidual sistêmica ${ }^{8}$. Alterações ultraestruturais pulmonares com aumento de peróxidos lipídicos e proteínas carboniladas.

A SOD é encontrada em grande quantidade nas vias aéreas e o glutatião, que exerce a mesma tarefa da catalase, pode ser encontrado no pulmão em proporção 100 vezes maior do que no plasma. No espaço intracelular localizam-se não só a SOD como a catalase armazenada em peroxissomos.

A produção de catalase nos eventos oxidativos e a proteção celular dependem da quantidade de $\mathrm{H}_{2} \mathrm{O}_{2}$ que chega a mitocôndria e da rapidez com que são gerados estes radicais e desta reserva anti-oxidante que se encontra em baixas concentrações neste órgão. Vários grupos de pesquisa já demonstraram que a administração de catalase na mitocôndria oferece proteção melhor do que a encontrada, habitualmente no citosol, quando existem lesões induzidas por $\mathrm{H}_{2} \mathrm{O}_{2} 9,26$.

Nos preparados histológicos foi possível observar a presença de neutrófilos, de fenômenos de congestão vascular, além da formação de atelectasias. Tais achados podem ser atribuídos à ação de oxirradicais, mediadores humorais com ação microcirculatória e citocina (TNF alfa) estimuladora da migração neutrofílica e que, por sua vez, amplifica a produção de espécies reativas do oxigênio e desencadeiam o dano pulmonar encontrado ${ }^{8}$.

Ressalte-se que, os polimorfonucleares do tipo neutrófilo, também produzem oxirradicais e, provavelmente, em tempo maior de observação já seria possível constatar o esgotamento desta reservas com a presença de lesões mais intensas. Lesões de reperfusão também podem ocorrer na ausência dos neutrófilos. DEEB e colaboradores estudaram lesão de pulmões isolados de ratos, isquemiados por 45 minutos e reperfundidos por 30 minutos, e observaram que a lesão foi inibida pela adição da catalase, mas não pela reperfusão com uma solução sem neutrófilos ${ }^{23}$. Isto evidencia a existência de mecanismos autóctones so- brepostos, geradores de espécies reativas do oxigênio.

Os mecanismos das lesões pulmonares de isquemia-reperfusão guardam pontos comuns com lesões de reperfusão em outros órgãos, a exemplo do intestino delgado, com participação significativa dos radicais livres do oxigênio, influxo intracelular de $\mathrm{Ca}_{2}{ }^{+}$, sequestro e ativação de leucócitária na circulação pulmonar, liberação de mediadores humorais, culminando com a lesão celular ${ }^{8,27}$.

Evidências na participação das espécies reativas do oxigênio na lesão pulmonar por l/R à distância motivaram vários estudos com a utilização de medidas terapêuticas antioxidantes de proteção ${ }^{8,10,28}$. Greca e colaboradores avaliaram, com sucesso, a utilização do azul de metileno como inibidor da produção de radicais superóxidos por agir como um receptor alternativo de elétrons provenientes da reação enzimática catalisada pela xantina oxidase ${ }^{29}$. O tratamento com propofol também se mostrou eficiente sendo capaz de minimizar as lesões de isquemia e reperfusão mesentérica em ratos $^{23}$. A n-acetilcisteína, com ações farmacológicas análogas a catalase, também foi eficiente na proteção antioxidante contra o estresse oxidativo produzido pela l/R intestinal ${ }^{24}$. Ainda são poucos os estudos clínicos que avaliam os benefícios de medidas terapêuticas para a lesão pulmonar de isquemia e reperfusão de tecidos localizados à distância ${ }^{8}$ e trata-se de área promissora para estudos futuros.

No rim duas importantes enzimas oxidativas são responsáveis pela geração de superóxido: a xantina oxidase (XO) e a nicotinamida adenina dinucleotídio fosfato (NADPH) oxidase $^{13}$. Considerando o aparecimento precoce de RLDO na lesão renal, aventa-se que estes oxirradicais estão diretamente relacionados à gênese da insuficiência deste órgão.

O comprometimento renal vinculado ao estresse oxidativo pode ser parcialmente atribuído ao acúmulo de ferro intrarrenal e que atua na formação do radical hidroxila, via reação de Haber-Weiss/Fenton ${ }^{30}$. A presença de uma reação oxidativa pode ser causada por lesões autóctones, mas eventualmente de condições à distância, não só por alterações homeostáticas, processos inflamatórios e infecciosos, como pela produção de mediadores que irão agir sobre o glomérulo, células tubulares ou a função renal.

Alguns autores já demonstraram que o pré-condicionamento intestinal é capaz de atenuar as lesões por isquemia e reperfusão renal, inclusive com aumento da atividade da catalase e de outros inativadores (SOD e glutatião peroxidase) ${ }^{31,32}$

A insuficiência renal crônica, também é associada com o estresse oxidativo, mas o mecanismo preciso carece, ainda, de elucidação. Estudos evidenciaram que a catalase e o glutatião peroxidase são sistemas eficazes de defesa porquanto catalisam a inativação do peróxido de hidrogênio e de outros hidroperóxidos orgânicos. Estudo em ratos, associado à nefrectomia 5/6, evidenciaram que ambas as enzimas são encontradas no rim e na aorta torácica. A catalase reduz-se acentuadamente nos animais submetidos à nefrectomia, por dano causado ao sistema de defesa antioxidante ${ }^{12}$. 
O ato operatório e a ação de oxirradicais desencadeiam vasoconstrição dos glomérulos do córtex renal e derivação do sangue para os néfrons da medula renal (reação de estresse), com potencial comprometimento parenquimatoso. Observaram-se nas amostras retiradas, congestão glomerular e declínio da atividade catalásica. Uma explicação para estes resultados, aparentemente pa- radoxais, reside na possibilidade do esgotamento precoce das reservas antioxidantes renais, em face do estímulo inicial, mas capaz de conter dano mais acentuado. Provavelmente, com maior tempo de isquemia intestinal, haveria maior produção de RLDO e outros mediadores (citocinas, proteínas de adesão e peróxidos lipídicos) e o dano parenquimatoso seria mais grave.

\section{A B S T R A C T}

Objective: This study aimed to assess the catalase activity after ischemia and reperfusion and to study the changes of this antioxidant in organs located far from the initial insult. Methods: Eighteen Wistar rats were randomly divided into three groups. 1Control, 2-Simulation and 3-Ischemia and Reperrfusion. In the latter it was done an ischemia of the ileum for 60 minutes followed by reperfusion for 30 minutes. In group 2 only laparotomy was performed. From all animals it was taken segments of the reperfused and non reperfused intestine, as well of the right kidney and lung to be evaluated under light microscopy. Catalase activity was measured in spectrophotometer with a wavelength set to $240 \mathrm{~nm}$. It was used Mann Whitney and Kruskal Wallis statistical tests. Results: There was a significant increase $(p<0.05)$ in the catalase activity not only at small bowel ischemic and non-ischemic segments but also at lungs. However the enzymatic activity decreases in the kidney. In all organs studied at reperfusion group it was found a slight villi derangement, mild congestion and infiltration with inflammatory cells, and areas of pulmonary atelectasis. Conclusion: The intestinal oxidative stress in rats causes biochemical changes at distance, with mobilization of antioxidant defense mechanisms in lung, non-ischemic intestinal segment and kidney, with early decrease in this last organ, however, with no relevant cellular damage.

Key words: Intestine, small. Catalase. Lung. Kidney. Rats.

\section{REFERENCIAS}

1. Ciz M, Cizova A, Chevion M, Lojek A, Kubala I, Papezikova I. Ischemia-reperfusion injury of rat small intestine: The effect of allopurinol dosage. Transplant Proc, 2001;33: 2871-3.

2. Thompson A, Hemphill D, Jeejeebhoy KN. Oxidative stress and antioxidants in intestinal disease. Dig Dis. 1998;16:152-8.

3. Souza DGS, Vieira AT, Pinho V, Sousa LP, Andrade AA, Bonjardim CA, Mcmilan M, Kahn M, Teixeira MM. NF-êB plays a major role during the sistemic and local acute inflammatory response following intestinal reperfusion injury. Br J Farmacol. 2005;145(2):246-54.

4. Junior OCS, Centurion S, Pacheco EG, Brisotti JL, Oliveira AF, Sasso $\mathrm{KD}$. Aspectos básicos da lesão de isquemia e reperfusão e do précondicionamento isquêmico. Acta Cir. Bras. 2002;17(3):96-100.

5. Forsyth, SF; Guilford, WG. Ischaemia-reperfusion injury.A small animal perspective. Brit. Vet. J. 1995;151:281-98.

6. Turan A, Gill R, Dudeja PK, Mohan H, Mahmood A. Effect of fat feeding on pro-oxidant and anti-oxidant enzyme systems in rat intestine: possible role in the turnover of enterocytes. Dig Dis Sci. 2009 Jun;54(6):1229-36.

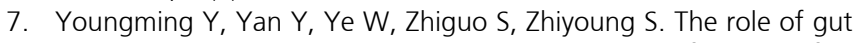
as a cytokine-generating organ in remote organ disfunction after intestinal ischemia and reperfusion. Chin Med J. 1998; 111(6): 514-8.

8. Pinheiro BV, Holanda MA, Araújo FG, Romaldini H. Lesão pulmonar de reperfusão. J Pneumol 1999;25(2):124-36.

9. Arita Y, Harkness SH, Kazzaz JA, Koo HC, Joseph A, Melendez JA, Davis JM, Chander A, And LI Y. Mitochondrial localization of catalase provides optimal protection from h2o2-induced cell death in lung epithelial cells. Am J Physiol Lung Cell Mol Physiol. 2006;290(5):L97886.

10. Zmijewski JW, Lorne E, Banerjee S, Abraham E. Participation of mitochondrial respiratory complex iii in neutrophil activation and lung injury. Am J Physiol Lung Cell Mol Physiol. 2009;296(4):L62434.

11. Iglesias JL, Lanoue JL, Rogers TE, Inman L, Turnage RH. Physiologic basis of pulmonary edema during intestinal reperfusion. J Surg Res. 1998;80:156-63.
12. Ram K. Sindhu, Ashkan Ehdaie, Farbod Farmand, Kanwaljit K. Dhaliwal, Tri Nguyen, Chang-De Zhan, Christian K. Roberts And Nosratola D. Vaziri. Expression of catalase and glutathione peroxidase in renal insufficiency. Biochim Biophys Acta 2005;1743: 86-92.

13. Yang CC, Ma MC, Chien CT, Wu MS, Sun WK, Chen CF. Hypoxic preconditioning attenuates lipopolysaccharide-induced oxidative stress in rat kidneys. J Physiol. 2007 1;582(Pt 1):407-19.

14. Paterno $F$, Longo WE. The etiology and pathogenesis of vascular disorders of the intestine. Radiol Clin North Am. 2008;46:877-85.

15. Rolim, MF.; Eleutherio, CA. E.; Eleutherio EC, Colão CDA F, Pereira GC, Schanaider A. Colonic healing after portal ischemia and reperfusion: an experimental study with oxidative stress biomarkers. Redox Rep. 2007;12: 267-74.

16. Schanaider A, Silva PC. Uso de animais em cirurgia experimental. Acta Cir. Bras.2004;19(4):441-7.

17. Schanaider A, Menezes VJMT, Borchardt AC, Oliveira PI, Madi K. Oxidized tissue proteins after intestinal reperfusion injury in rats. Acta Cir Bras. 2005;20(6):434-6.

18. Cerqueira NF, Hussni CA, Yoshida WB. Pathophysiology of mesenteric ischemia/reperfusion: a review. Acta Cir Bras. 2005;20(4):336-43.

19. Brito MVH, Araújo M, Acácio GJS, Acácio GJS, Reis JMC. Lesão intestinal após isquemia-reperfusão: estudo comparativo usando sal tetrazólico (mtt) e histologia. Acta Cir. Bras. 2001;16(1):26-31

20. Khanna A, Rossman JE, Fung HI, Caty MG. Intestinal and hemodynamic impairment following mesenteric ischemia/ reperfusion. J Surg Res. 2001;99:114-9.

21. Parks DA, Bulkley GB, Granger DN. Role of oxygen-derived free radicals in digestive tract diseases. Surgery. 1983;94(3):415-22.

22. Caty MG, Guice KS, Oldham KT, Remick DG, Kunkel SI. Evidence for tumor necrosis factor-induced pulmonary microvascular injury after intestinal ischemia-reperfusion injury. Ann Surg. 1990;212(6):694-700.

23. Santos CHM, Gomes OM, Pontes JCDV, Vieira JCDV, Miiji LNO, Higa El. Use of propofol (2,6 diisopropilphenol) as inhibitor of tissue lesion in mesenteric ischemia and reperfeusion: experimental studud in rats. Acta Cir Bras. 2003, 18: 347-54. 
24. Azeredo M, Azeredo L, Eleuthério E, Schanaider A. Propofol and $n$-acetylcysteine attenuate oxidative stress induced by intestinal ischemia/reperfusion in rats. Protein carbonyl detection by immunoblotting. Acta Cir Bras. 2008;23(5). 425-8.

25. Gerkin TM, Oldham KT, Guice KS, Hinshaw DB, Ryan US. Intestinal ischemia-reperfusion injury. Causes pulmonary endothelial cell ATP depletion. Ann Surg. $1993 ; 217(1): 48-56$

26. Salvi M, Battaglia V, Brunati AM, La Rocca N, Tibaldi E, Pietrangeli $\mathrm{P}$, Marcocci L, Mondovì B, Rossi CA, Toninello A. Catalase takes part in rat liver mitochondria oxidative stress defense. J Biol Chem. 2007; 17;282(33):24407-15.

27. Liu KX, Chen SQ, Zhang H, Guo JY, Li YS, Huang WQ. Intestinal ischaemia/reperfusion upregulates beta-defensin-2 expression and causes acute lung injury in the rat. Injury. 2009;40(9):950-5.

28. Osborne DI, Aw TY, Cepinskas G, Kvietys PR. Development of ischemia/reperfusion tolerance in rat small intestine. J Clin Invest. $1994 ; 94(5): 1910-8$.

29. Greca FH, Gonçalves NMFM, Filho ZAS, Silva APG, Mima WH, Mima $\mathrm{HH}$. The protective effect of methylene blue in lungs, small bowel and kidney after intestinal ischemia and reperfusion. Acta Cir. Bras. 2008;23(2):149-56.

30. Paller MS, Hoidal JR, Ferris TF. Oxygen free radicals in ischemic acute renal failure in the rat. J Clin Invest. 1984; 74:1156-64.
31. Song $T$, Peng YF, Guo SY, Liu YH, Liul LY. Brief small intestinal ischemia lessens renal ischemia-reperfusion injury in rats. Comp Med. 2007;57(2):200-5.

32. Yun Y, Duan WG, Chen P, Wu HX, Shen ZQ, Qian ZY, Wang DH. Ischemic postconditioning modified renal oxidative stress and lipid peroxidation caused by ischemic reperfusion injury in rats. Transplant Proc. 2009;41(9):3597-602.

Recebido em 07/01/2009

Aceito para publicação em 09/03/2009

Conflito de interesse: nenhum

Fonte de financiamento: FAPERJ

\section{Como citar este artigo:}

Ferro CO, Chagas VLA, Oliveira MF, Oliveira PL, Schanaider A. Atividade da catalase no pulmão, rim e intestino delgado não isquemiado de ratos após reperfusão intestinal. Rev Col Bras Cir. [periódico na Internet] 2010; 37(1). Disponível em URL: http://www.scielo.br/rcbc

\section{Endereço para correspondência:}

Camila Oliveira

E-mail: camyoliva@yahoo.com.br 